\title{
A multiplicative version of the Lindley recursion
}

\author{
Onno Boxma ${ }^{1} \cdot$ Andreas Löpker $^{2} \cdot$ Michel Mandjes $^{3} \cdot$ Zbigniew Palmowski $^{4}$
}

Received: 3 March 2020 / Revised: 9 February 2021 / Accepted: 12 February 2021 /

Published online: 13 March 2021

(c) The Author(s) 2021

\begin{abstract}
This paper presents an analysis of the stochastic recursion $W_{i+1}=\left[V_{i} W_{i}+Y_{i}\right]^{+}$that can be interpreted as an autoregressive process of order 1 , reflected at 0 . We start our exposition by a discussion of the model's stability condition. Writing $Y_{i}=B_{i}-A_{i}$, for independent sequences of nonnegative i.i.d. random variables $\left\{A_{i}\right\}_{i \in \mathbb{N}_{0}}$ and $\left\{B_{i}\right\}_{i \in \mathbb{N}_{0}}$, and assuming $\left\{V_{i}\right\}_{i \in \mathbb{N}_{0}}$ is an i.i.d. sequence as well (independent of $\left\{A_{i}\right\}_{i \in \mathbb{N}_{0}}$ and $\left\{B_{i}\right\}_{i \in \mathbb{N}_{0}}$ ), we then consider three special cases (i) $V_{i}$ equals a positive value $a$ with certain probability $p \in(0,1)$ and is negative otherwise, and both $A_{i}$ and $B_{i}$ have a rational LST, (ii) $V_{i}$ attains negative values only and $B_{i}$ has a rational LST, (iii) $V_{i}$ is uniformly distributed on $[0,1]$, and $A_{i}$ is exponentially distributed. In all three cases, we derive transient and stationary results, where the transient results are in terms of the transform at a geometrically distributed epoch.
\end{abstract}

Keywords Lindley recursion · Autoregressive models · Wiener-Hopf boundary value problem - Laplace transform

Zbigniew Palmowski

zbigniew.palmowski@pwr.edu.pl

Onno Boxma

o.j.boxma@tue.nl

Andreas Löpker

lopker@htw-dresden.de

Michel Mandjes

m.r.h.mandjes@uva.nl

1 Eurandom and the Department of Mathematics and Computer Science, Eindhoven University of Technology, P.O. Box 513, 5600 MB Eindhoven, The Netherlands

2 HTW Dresden, University of Applied Sciences, Friedrich-List-Platz 1, 01069 Dresden, Germany

3 Korteweg-de Vries Institute for Mathematics, University of Amsterdam, Science Park 904, 1098 XH Amsterdam, The Netherlands

4 Department of Applied Mathematics, Faculty of Pure and Applied Mathematics, Wrocław University of Science and Technology, Wyb. Wyspiańskiego 27, 50-370 Wrocław, Poland 
Mathematics Subject Classification Primary: 60K25; Secondary: 90B22

\section{Introduction}

This paper focuses on the Lindley type stochastic recursion

$$
W_{i+1}=\left[V_{i} W_{i}+Y_{i}\right]^{+}, \quad i=0,1, \ldots,
$$

where $[x]^{+}=\max \{x, 0\}$ for $x \in \mathbb{R}$, and $\left\{V_{i}\right\}_{i \in \mathbb{N}_{0}}$ and $\left\{Y_{i}\right\}_{i \in \mathbb{N}_{0}}$ are independent sequences of i.i.d. (independent, identically distributed) random variables. The analysis of stochastic recursions has received much attention in the applied probability literature. This holds in particular for stochastic recursions of the autoregressive type, owing to their wide applicability across various scientific domains including biology, finance, and engineering $[7,9,14]$.

An important subclass of first-order autoregressive models corresponds to the case in which the $\left\{V_{i}\right\}_{i \in \mathbb{N}_{0}}$ are constant, i.e., a stochastic process defined through the recursion

$$
W_{i+1}=a W_{i}+Y_{i}, \quad i=0,1, \ldots,
$$

for a sequence of i.i.d. random variables $\left\{Y_{i}\right\}_{i \in \mathbb{N}_{0}}$ and a scalar $a$, with $W_{0}$ being given. When the quantities $W_{i}$ cannot attain negative values, it becomes natural to study the truncated counterpart of (2), i.e., the recursion

$$
W_{i+1}=\left[a W_{i}+Y_{i}\right]^{+}, \quad i=0,1, \ldots
$$

When $a=1$ we recover the classical Lindley recursion describing the waiting time in the G/G/1 queue, with $Y_{i}$ representing the difference between the $i$-th service time and the $(i+1)$-st interarrival time. The case of $a \in(0,1)$ was studied in detail in [7], whereas the case $a=-1$ is covered by [23]. It should be observed that, while from the analysis it is clear that $a$ is assumed to be positive in [7], the introduction of that paper incorrectly states that $|a|<1$.

By studying (1), we significantly extend the analysis of the Lindley recursion as well as the analysis of the stochastic recursion (3). Our results focus on three different choices of $\left\{V_{i}\right\}$. In Model I, the $V_{i}$ are either negative or equal to the positive constant $a$. Here, we demand that both the positive and the negative parts of the $Y_{i}$ have a rational Laplace-Stieltjes transform (in the sequel abbreviated to LST). In Model II, the $V_{i}$ are negative random variables. A detailed analysis is shown to be possible as long as the positive part of the $Y_{i}$ has a rational LST. While Model I to a large extent contains Model II, we prefer to give a separate analysis of both models, to make the reader familiar with the specific mathematical intricacies due to $V_{i}$ being negative (Model II) and $V_{i}$ being a positive constant (the model in [7]). Moreover, the extra assumption that the negative part of the $Y_{i}$ has a rational LST plays an important part in the analysis of Model I, precluding the possibility to simply obtain the results for Model II from those for Model I. Finally, in Model III, the $V_{i}$ are uniformly distributed 
on $[0,1]$, and the negative part of the $Y_{i}$ is exponentially distributed; this case requires an entirely different approach.

The rationality assumptions are natural in the light of the existing theory that has been developed for the G/G/1 queue. While in principle the waiting-time distribution in the general G/G/1 queue can be obtained via a Wiener-Hopf decomposition (cf. [11, Chapter II.5]), the solution is a rather implicit one, unless one makes rationality assumptions on either the interarrival or the service-time LST. In addition, it can be argued that the distribution of any nonnegative random variable can be approximated arbitrarily closely by the distribution of a random variable with a rational LST [1, Ch. III], so that a restriction to random variables with rational LST leads to just a minor loss of generality.

Notable studies of stochastic recursions are $[4,13,16]$; see in addition [3]. For the non-reflected case, stochastic recursions of the form $W_{i+1}=V_{i} W_{i}+Y_{i}$ have been studied frequently, partly under the name 'Vervaat perpetuity'; we mention $[9,12,14$, 17,19,22]. For the reflected case, [8] considers another generalization of the Lindley recursion, by replacing $V_{i} W_{i}$ in (1) by $S\left(W_{i}\right)$, where $\{S(t)\}_{t \geq 0}$ is a Lévy subordinator. A model that is similar to the present model has been discussed in [24]. It is noted, though, that [24] primarily focuses on stability questions, limit theorems and questions related to queuing applications, whereas our primary focus lies on the derivation of results for the transient and stationary distribution of the process under investigation. Also related is the model in [6]; there (1) is considered with $\mathbb{P}\left(V_{i}=1\right)=p, \mathbb{P}\left(V_{i}=-1\right)=1-p$.

The main contributions of the present paper are the following: For Models I and II, we state and solve a Wiener-Hopf boundary value problem, which allows us to study the transient behavior of the $\left\{W_{i}\right\}_{i \in \mathbb{N}_{0}}$ process. In particular, we obtain an expression for the object

$$
\sum_{i=0}^{\infty} r^{i} \mathbb{E}\left(\mathrm{e}^{-s W_{i}}\right)
$$

which can be interpreted as the generating function of the LST of the $W_{i}$, but also (up to the multiplicative constant $1-r)$ as the LST after a geometrically distributed time. The transient behavior of the $\left\{W_{i}\right\}_{i \in \mathbb{N}_{0}}$ process is also obtained for Model III, using a different argument. The stability condition of each of the three models is discussed, and the steady-state distribution of the $\left\{W_{i}\right\}_{i \in \mathbb{N}_{0}}$ process is also determined.

The remainder of the paper is organized as follows: Section 2 presents the description of the three models and some preliminaries. Sections 3, 4 and 5 are devoted to the transient and steady-state analysis of, respectively, Models I, II, and III. Section 6 contains a discussion and provides suggestions for further research.

\section{Model description and preliminaries}

The main object of study is the stochastic recursion

$$
W_{i+1}=\left[V_{i} W_{i}+Y_{i}\right]^{+}, \quad i=0,1, \ldots,
$$


where $\left\{V_{i}\right\}_{i \in \mathbb{N}_{0}}$ and $\left\{Y_{i}\right\}_{i \in \mathbb{N}_{0}}$ are sequences of i.i.d. random variables, which are in addition independent of each other. The initial state of the process is assumed to be $W_{0}=w \in \mathbb{R}^{+}$. We write $V$ and $Y$ for generic random variables distributed as $V_{0}$ and $Y_{0}$ respectively.

In this paper, we discuss the following three variants of the model:

$$
\begin{aligned}
& \text { ModelI : } \mathbb{P}(V=a)=p, \mathbb{P}(V<0)=1-p, \quad a>0, p \in(0,1) \\
& \text { Model II : } \mathbb{P}(V<0)=1 ; \\
& \text { Model III : } \mathbb{P}(V<x)=x, \quad 0 \leq x \leq 1 .
\end{aligned}
$$

In each of the cases, we will assume that the $Y_{i}$ are decomposed as $B_{i}-A_{i}$, with sequences $\left\{A_{i}\right\}_{i \in \mathbb{N}_{0}}$ and $\left\{B_{i}\right\}_{i \in \mathbb{N}_{0}}$ of i.i.d. nonnegative random variables. In addition, depending on the chosen model, the random variables $A_{i}$ and/or $B_{i}$ are assumed to have a rational LST.

We start with investigating the stationary behavior of $\left\{W_{i}\right\}_{i \in \mathbb{N}_{0}}$. We always assume that both $\mathbb{E}|V|$ and $\mathbb{E}|Y|$ are finite. The first result was given in [24] and covers most cases of interest.

Theorem 1 [24] If one of the following conditions holds, then $W_{i}$ tends weakly to a proper limit $W$ as $i \rightarrow \infty$ :

(C1) $\mathbb{P}(V<0)>0$ and $\mathbb{P}(Y \leq 0)>0$,

(C2) $V \geq 0$ a.s. and $\mathbb{P}(V=0)>0$,

(C3) $V>0$ a.s. and $\mathbb{E}(\log |V|)<0$.

Moreover, $W_{i}$ converges weakly to a possibly improper limit $W$ as $i \rightarrow \infty$ if $V>0$ a.s., $\mathbb{E}(\log |V|)=0$, and $W_{0}=0$. If additionally $V=1$ a.s. then $W$ is proper for $\mathbb{E}(Y)<0$ and improper for $\mathbb{E}(Y)>0$.

It follows straightforwardly from the regenerative structure of $W_{i}$, and the proof of the above theorem in [24], that in cases (C1) and (C2) the limit $W$ is unique. Obviously, under the conditions of the theorem, the limiting random variable $W$ fulfils the associated distributional identity $W={ }_{\mathrm{d}}[V W+Y]^{+}$. Regarding the above condition (C3), we add the following observation.

Theorem 2 In order to have convergence of $W_{i}$ to a proper unique limit $W$ as $i \rightarrow \infty$, it is sufficient to have $\mathbb{E}(\log |V|)<0$, which in turn is implied by $\mathbb{E}|V|<1$.

Proof Recursion (4) can be written as a random iteration $W_{i+1}=f_{\theta_{i}}\left(W_{i}\right)$ with $f_{\theta_{i}}(x)=\left[x V_{i}+Y_{i}\right]^{+}, \theta_{i}=\left(V_{i}, Y_{i}\right)$. This means that $f_{\theta}(\cdot)$ enjoys the Lipschitz property

$$
\left|f_{\theta}(x)-f_{\theta}(y)\right| \leq K_{\theta}|x-y|
$$

with random Lipschitz constant $K_{\theta}=|V|$. As a result, [13, Thm. 1.1] is applicable. By Jensen's inequality, $\mathbb{E} \log |V| \leq \log \mathbb{E}|V|$ and so $\mathbb{E}|V|<1$ implies the condition $\mathbb{E}(\log |V|)<0$. 
The case where $\mathbb{P}(V<0)>0$ and $Y \geq 0$ a.s., which was omitted in [24], is more involved due to the fact that the process might not be aperiodic, even if $Y$ is not deterministic. As an example suppose that the distribution of $Y$ is supported on $[1,2]$ and that $V \leq-2$ a.s. If $W_{0}=0$, then $W_{1} \in[1,2], W_{2}=0, W_{3} \in[1,2]$, entailing that the process alternates between the set $\{0\}$ and a value in $[1,2]$. On the other hand, if $W_{0}>2$, then $W_{1}=0, W_{2} \in[1,2]$ and so on. As a consequence, there is no convergence $W_{i} \Rightarrow W$ as $i \rightarrow \infty$. However, regarding the existence of a stationary distribution, we can show the following.

Theorem 3 If $\mathbb{P}(V \leq 0)>0$ and $Y \geq 0$ a.s., then there is convergence of $W_{i}$ to a stationary random variable $W$ as $i \rightarrow \infty$.

Proof We define a majorizing process by $M_{0}:=W_{0}$ and $M_{i+1}:=\left[V_{i}\right]^{+} M_{i}+Y_{i}$. Then, $W_{i} \leq M_{i}, i=0,1,2, \ldots$ and for $\left\{M_{i}\right\}_{i \in \mathbb{N}_{0}}$ we have $\mathbb{E}\left(\log \left|[V]^{+}\right|\right)=-\infty<0$ since $\mathbb{P}\left([V]^{+}=0\right)>0$. So by Theorem 2 it follows that $M_{i} \Rightarrow M$ as $i \rightarrow \infty$ for some limiting random variable $M$. Hence, $\left\{M_{i}\right\}_{i \in \mathbb{N}_{0}}$ is tight, and then, the sequence $\left\{W_{i}\right\}_{i \in \mathbb{N}_{0}}$ is tight as well. Thus, [15, Thm. 4] guarantees the existence of a stationary distribution, for $\left\{W_{i}\right\}_{i \in \mathbb{N}_{0}}$.

We end this section with a lemma that forms the starting-point of the analysis of all three models. For this, we need to introduce some additional notation. For a given nonnegative random variable $X$, we write $\Phi_{X}(s)=\mathbb{E} e^{-s X}$ for its LST, defined at least for $\operatorname{Re} s \geq 0$. We say that $\Phi_{X} \in \mathbb{Q}\left[s_{1}, s_{2}, \ldots, s_{n}\right]$ if $X$ has a rational LST with poles at $s_{1}, s_{2}, \ldots, s_{n}$, i.e., if $\Phi_{X}(s)$ is of the form

$$
\Phi_{X}(s)=\frac{N_{X}(s)}{D_{X}(s)},
$$

where $D_{X}(s)=\prod_{i=1}^{n}\left(s-s_{i}\right)$ and $N_{X}(s)$ is a polynomial of degree at most $n-1$ not sharing zeros with $D_{X}(s)$. Note that this implies that $\mathbb{P}(X=0)=\lim _{s \rightarrow \infty} \Phi_{X}(s)=$ 0 . With this notation we then have, for example, $\Phi_{Y}(s)=\Phi_{B}(s) \Phi_{A}(-s)$. We also write $D_{Y}(s)=D_{B}(s) D_{A}(-s)$ and $N_{Y}(s)=N_{B}(s) N_{A}(-s)$ if $A$ and $B$ have rational LSTs of the form (5).

For a sequence $\left\{X_{i}\right\}_{i \in \mathbb{N}_{0}}$ of random variables, we introduce the generating function, for $r \in(0,1)$ :

$$
U_{X}(r, s)=\sum_{i=0}^{\infty} r^{i} \Phi_{X_{i}}(s) .
$$

Note that since $\Phi_{V X}(s)=\int \Phi_{X}(y s) \mathbb{P}(V \in \mathrm{d} y)$, we have

$$
U_{V X}(r, s)=\int U_{X}(r, y s) \mathbb{P}(V \in \mathrm{d} y) .
$$

The following lemma plays a key role in our analysis. Define $W_{i}^{*}:=\left[V_{i} W_{i}+Y_{i}\right]^{-}$, where $[x]^{-}:=\min \{x, 0\}$. 
Lemma $4 U_{W}(r, s)$ and $U_{W^{*}}(r, s)$ are, for $r \in(0,1)$ and $\operatorname{Re} s=0$, related via

$$
U_{W}(r, s)=\mathrm{e}^{-s w}+r\left(\Phi_{Y}(s) U_{V W}(r, s)+\frac{1}{1-r}-U_{W^{*}}(r, s)\right) .
$$

Proof First observe that the basic identity $\exp \left([x]^{+}\right)=\exp (x)+1-\exp \left([x]^{-}\right)$ applies. It thus follows from (4) that

$$
\Phi_{W_{i+1}}(s)=\Phi_{V_{i} W_{i}+Y_{i}}(s)+1-\Phi_{W_{i}^{*}}(s), \quad i=0,1, \ldots
$$

Multiplying both sides of (8) by $r^{i+1}$ and summing yields the identity (7).

\section{Model I: the mixed case}

In this section, we consider the following model: We start from the recursion (4), and we assume that $V=a$ for $a>0$ with probability $p$ and $V<0$ with probability $1-p$. Let

$$
V^{-}=(V \mid V<0)
$$

We assume that both $A$ and $B$ have a rational LST. Summarizing, we impose the conditions
(A) $\mathbb{P}(V=a)=p, \mathbb{P}(V<0)=1-p, a>0, p \in(0,1)$,
(B) $\Phi_{B} \in \mathbb{Q}\left[s_{1}, \ldots, s_{\ell}\right]$ with $\operatorname{Re} s_{j}<0$ for $j=1, \ldots, \ell$,
(C) $\Phi_{A} \in \mathbb{Q}\left[t_{1}, \ldots, t_{m}\right]$ with $\operatorname{Re} t_{i}<0$ for $i=1, \ldots, m$.

Theorem 5 Suppose that the conditions (A), (B), and (C) hold. Then, for $r \in(0,1)$,

1. if $a=1$ then

$$
U_{W}(r, s)=\frac{D_{Y}(s) \mathrm{e}^{-s w}+\sum_{k=0}^{m+\ell} a_{k}(r) s^{k}}{D_{Y}(s)-r p N_{Y}(s)}
$$

where

$$
a_{0}(r)=\frac{r}{1-r}(1-p)(-1)^{\ell+m} \prod_{j=1}^{\ell} s_{j} \prod_{i=1}^{m} t_{i}
$$

while the remaining constants $a_{1}(r), \ldots, a_{m+\ell}(r)$ can be determined from the linear systems (16) and (18) that will be given below.

2. if $a \neq 1$ then

$$
U_{W}(r, s)=\sum_{h=0}^{\infty}\left(\mathrm{e}^{-a^{h} s w}+\frac{\sum_{k=0}^{m+\ell} a_{k}(r)\left(a^{h} s\right)^{k}}{D_{Y}\left(a^{h} s\right)}\right)(r p)^{h} \prod_{j=0}^{h-1} \Phi_{Y}\left(a^{j} s\right),
$$


where $a_{0}(r)$ is as in (10) and the remaining constants $a_{1}(r), \ldots, a_{m+\ell}(r)$ can be determined from the linear systems (26) and (27) that will be given below.

Proof In this situation

$$
U_{V W}(r, s)=p U_{W}(r, a s)+(1-p) \int_{-\infty}^{0} U_{W}(r, s y) \mathbb{P}\left(V^{-} \in \mathrm{d} y\right) .
$$

Then (7) becomes, after multiplication by $D_{Y}(s)$,

$$
\begin{aligned}
& D_{Y}(s)\left(U_{W}(r, s)-\mathrm{e}^{-s w}\right)-r p N_{Y}(s) U_{W}(r, a s) \\
& \quad=r(1-p) N_{Y}(s) \int_{-\infty}^{0} U_{W}(r, s y) \mathbb{P}\left(V^{-} \in \mathrm{d} y\right)+r D_{Y}(s)\left(\frac{1}{1-r}-U_{W^{*}}(r, s)\right) .
\end{aligned}
$$

Now the following are true:

(i) the left-hand side of (13) is analytic in $\operatorname{Re} s>0$ and continuous in $\operatorname{Re} s \geq 0$,

(ii) the right-hand side of (13) is analytic in $\operatorname{Re} s<0$ and continuous in $\operatorname{Re} s \leq 0$,

(iii) for large $s$, both sides are $O\left(s^{m+\ell}\right)$ in their respective half-planes.

Both sides are well defined at the boundary $\operatorname{Re} s=0$. Determination of the unknown functions $U_{W}(r, s)$ and $U_{W^{*}}(r, s)$ from (13) and conditions (i), (ii), and (iii) is a Wiener-Hopf boundary value problem of a type that has been extensively studied in queueing theory before, cf. the expository paper [10]. By introducing a function $G(r, s)$ that is equal to the left-hand side of (13) for $\operatorname{Re} s \geq 0$ and to the right-hand side of (13) for $\operatorname{Re} s \leq 0$, we have a function that is analytic in the whole $s$-plane, and that for large $s$ is $O\left(s^{m+\ell}\right)$. Liouville's theorem [21, p. 85] now states that both sides of (13), in their respective half-planes, are equal to the same $(m+\ell)$-th degree polynomial in $s$. In other words, for $\operatorname{Re} s \geq 0$,

$$
D_{Y}(s)\left(U_{W}(r, s)-\mathrm{e}^{-s w}\right)-r p N_{Y}(s) U_{W}(r, a s)=\sum_{k=0}^{m+\ell} a_{k}(r) s^{k},
$$

and, for $\operatorname{Re} s \leq 0$,

$$
\begin{aligned}
& r(1-p) N_{Y}(s) \int_{-\infty}^{0} U_{W}(r, s y) \mathbb{P}\left(V^{-} \in \mathrm{d} y\right)+r D_{Y}(s)\left(\frac{1}{1-r}-U_{W^{*}}(r, s)\right) \\
& \quad=\sum_{k=0}^{m+\ell} a_{k}(r) s^{k} .
\end{aligned}
$$

Taking $s=0$ in either (14) or (15) yields, after a straightforward calculation, the expression for $a_{0}(r)$ in (10). Next we set $s=s_{j}, j=1, \ldots, \ell$, in (15). Since $D_{B}\left(s_{j}\right)=$ 
0 it follows that

$$
r(1-p) N_{Y}\left(s_{j}\right) \int_{-\infty}^{0} U_{W}\left(r, s_{j} y\right) \mathbb{P}\left(V^{-} \in \mathrm{d} y\right)=\sum_{k=0}^{m+\ell} a_{k}(r) s_{j}^{k}, \quad j=1, \ldots, \ell .
$$

We thus have obtained $\ell$ linear equations in the remaining $m+\ell$ unknown $a_{k}(r)$; however, they are expressed in the yet unknown function $U_{W}(r, \cdot)$.

We turn to (14), which provides a relation between $U_{W}(r, s)$ and $U_{W}(r, a s)$. As it turns out, we have to distinguish between the two cases $a=1$ and $a \neq 1$ :

○ Case $i$ : For $a=1$, after division by the denominators, Relation (14) can be rewritten as

$$
U_{W}(r, s)\left(1-\operatorname{rp} \Phi_{Y}(s)\right)=\mathrm{e}^{-s w}+\frac{\sum_{k=0}^{m+\ell} a_{k}(r) s^{k}}{D_{Y}(s)}
$$

Cohen [11], in his study of the $\mathrm{K}_{m} / \mathrm{G} / 1$ queue, proves that the term between brackets in the left-hand side of (17) has $m$ zeroes $\delta_{1}(r), \ldots, \delta_{m}(r)$ in the right half plane $\operatorname{Re} s>0$. The analyticity of $U_{W}(r, s)$ for $\operatorname{Re} s \geq 0$ now implies that the righthand side of (17) must be zero for all these $m$ zeroes. This results in the $m$ linear equations

$$
\sum_{k=0}^{m+\ell} \delta_{i}^{k}(r) a_{k}(r)=-\mathrm{e}^{-\delta_{i}(r) w} D_{Y}\left(\delta_{i}(r)\right), \quad i=1, \ldots, m
$$

Formula (16) contains $\ell$ more equations in the $a_{k}(r)$. Relying on (17), we can rewrite it into

$r(1-p) N_{Y}\left(s_{j}\right) \int_{-\infty}^{0} \frac{\mathrm{e}^{-s_{j} y w}+\sum_{k=0}^{m+\ell} a_{k}(r) \frac{\left(s_{j} y\right)^{k}}{D_{Y}\left(s_{j} y\right)}}{1-r p \Phi_{Y}\left(s_{j} y\right)} \mathbb{P}\left(V^{-} \in \mathrm{d} y\right)=\sum_{k=0}^{m+\ell} a_{k}(r) s_{j}^{k}$,

for $j=1, \ldots, \ell$. From this, we obtain

$$
\sum_{k=0}^{m+\ell} c_{k}\left(r, s_{j}\right) a_{k}(r)=\int_{-\infty}^{0} \frac{\mathrm{e}^{-s_{j} y w}}{1-r p \Phi_{Y}\left(s_{j} y\right)} \mathbb{P}\left(V^{-} \in \mathrm{d} y\right), \quad j=1,2, \ldots, \ell,
$$

where, for $j=1, \ldots, \ell$,

$$
c_{k}\left(r, s_{j}\right)=\frac{s_{j}^{k}}{r(1-p) N_{Y}\left(s_{j}\right)}-\int_{-\infty}^{0} \frac{\left(s_{j} y\right)^{k}}{D_{Y}\left(s_{j} y\right)-r p N_{Y}\left(s_{j} y\right)} \mathbb{P}\left(V^{-} \in \mathrm{d} y\right) .
$$


- Case ii: For $a<1$, Relation (14) has the same structure as [7, Formula (2.3)]. Proceeding in a similar way as in [7], we write

$$
U_{W}(r, s)=K(r, s) U_{W}(r, a s)+L(r, s),
$$

with

$$
K(r, s):=\operatorname{rp} \Phi_{Y}(s), \quad L(r, s):=\mathrm{e}^{-s w}+\frac{\sum_{k=0}^{m+\ell} a_{k}(r) s^{k}}{D_{Y}(s)}
$$

For $a \neq 1$ iteration of (21) yields

$$
U_{W}(r, s)=\sum_{h=0}^{\infty} L\left(r, a^{h} s\right) \prod_{j=0}^{h-1} K\left(r, a^{j} s\right)
$$

where convergence of the infinite sum can be proven using the d'Alembert test. Indeed, for $a<1$ the limit as $h \rightarrow \infty$ of the ratio of two successive terms is

$$
\lim _{h \rightarrow \infty}\left|\frac{L\left(r, a^{h} s\right)}{L\left(r, a^{h+1} s\right) K\left(r, a^{h} s\right)}\right|=\frac{1}{r p}>1,
$$

while for $a>1, K\left(r, a^{h} s\right) \rightarrow 0$ and $\left|L\left(r, a^{h} s\right)\right| \rightarrow a_{m+\ell}(r)$, causing divergence of the left-hand side in (24) to infinity.

Insertion of (22) in (23) gives (11). The only unknowns are $a_{1}(r), \ldots, a_{m+\ell}(r)$. We obtain $m$ linear equations in the unknown $a_{k}(r)$ by observing that substitution of $s=-t_{i}, i=1, \ldots, m$, in (14) results in the following identity:

$$
-r p N_{Y}\left(-t_{i}\right) U_{W}\left(r,-a t_{i}\right)=\sum_{k=0}^{m+\ell} a_{k}(r)\left(-t_{i}\right)^{k}, \quad i=1, \ldots, m
$$

Substituting the right-hand side of (11), with $s=-a t_{i}$, into (25) now gives, for $a \neq 1$, the $m$ linear equations

$$
\begin{aligned}
& \sum_{k=0}^{m+\ell}\left(-t_{i}\right)^{k} a_{k}(r)\left(1+\operatorname{rp} N_{Y}\left(-t_{i}\right) \sum_{h=0}^{\infty} \frac{a^{k(h+1)}(r p)^{h} \prod_{j=0}^{h-1} \Phi_{Y}\left(-a^{j+1} t_{i}\right)}{D_{Y}\left(-a^{h+1} t_{i}\right)}\right) \\
& \quad=-r p N_{Y}\left(-t_{i}\right) \sum_{h=0}^{\infty} \mathrm{e}^{a^{h+1} t_{i} w}(r p)^{h} \prod_{j=0}^{h-1} \Phi_{Y}\left(-a^{j+1} t_{i}\right)
\end{aligned}
$$


for $i=1, \ldots, m$. The remaining $\ell$ equations are provided by substituting (11) into (16), yielding

$$
\begin{aligned}
& \sum_{k=0}^{m+l} d_{k}\left(r, s_{j}\right) a_{k}(r) \\
& =r(1-p) N_{Y}\left(s_{j}\right) \sum_{h=0}^{\infty}(r p)^{h} \int_{-\infty}^{0} \mathrm{e}^{-a^{h} s_{j} y w} \prod_{i=0}^{h-1} \Phi_{Y}\left(a^{i} s_{j} y\right) \mathbb{P}\left(V^{-} \in \mathrm{d} y\right)
\end{aligned}
$$

for $j=1, \ldots, \ell$, where

$$
\begin{aligned}
& d_{k}\left(r, s_{j}\right) \\
& \quad=s_{j}^{k}\left(1-r(1-p) N_{Y}\left(s_{j}\right) \sum_{h=0}^{\infty}(r p)^{h} \int_{-\infty}^{0} \frac{\left(a^{h} y\right)^{k} \prod_{i=0}^{h-1} \Phi_{Y}\left(a^{i} s_{j} y\right)}{D_{Y}\left(a^{h} s_{j} y\right)} \mathbb{P}\left(V^{-} \in \mathrm{d} y\right)\right) .
\end{aligned}
$$

This finishes the proof.

The steady-state LST of $W$ exists if $\mathbb{P}(B \leq A)>0$, cf. Theorem 1 . It can be obtained by applying an Abelian theorem. For example in case (ii), in which $a<1$, one gets

$$
\Phi_{W}(s)=\lim _{r \uparrow 1}(1-r) U_{W}(r, s)=\sum_{h=0}^{\infty} L\left(a^{h} s\right) \prod_{j=0}^{h-1} K\left(a^{j} s\right),
$$

with

$$
K(s):=p \Phi_{Y}(s), \quad L(s):=\frac{\sum_{k=0}^{m+l} a_{k} s^{k}}{D_{Y}(s)}
$$

where $a_{k}:=\lim _{r \uparrow 1}(1-r) a_{k}(r)$.

\section{Model II: the negative case}

The model we analyze in this section assumes that each $V_{i}$ attains only negative values and that $Y_{i}$ is the difference $B_{i}-A_{i}$ of two independent nonnegative random variables, where $B_{i}$ has a rational LST. In other words, we impose the conditions

(A*) $V<0$ a.s.,

(B) $\Phi_{B} \in \mathbb{Q}\left[s_{1}, \ldots, s_{\ell}\right]$ with $\operatorname{Re} s_{j}<0$ for $j=1, \ldots, \ell$.

We no longer need to impose condition (C) regarding the rationality of $\Phi_{A}(\cdot)$.

Theorem 6 Suppose that the conditions $\left(\mathrm{A}^{*}\right)$ and $(\mathrm{B})$ hold. Then, for $r \in(0,1)$,

$$
U_{W}(r, s)=\mathrm{e}^{-s w}+\frac{\sum_{k=0}^{\ell} a_{k}(r) s^{k}}{D_{B}(s)}, \quad \operatorname{Re} s \geq 0,
$$


where

$$
a_{0}(r)=\frac{r}{1-r}(-1)^{\ell} \prod_{j=1}^{\ell} s_{j},
$$

and the remaining constants $a_{1}(r), \ldots, a_{\ell}(r)$ can be determined from the linear system (39) that will be given below.

Proof Multiplying both sides of (7) by the denominator $D_{B}(s)$ gives

$$
\begin{aligned}
& D_{B}(s)\left(U_{W}(r, s)-\mathrm{e}^{-s w}\right) \\
& \quad=r N_{B}(s) \Phi_{A}(-s) U_{V W}(r, s)+r D_{B}(s)\left(\frac{1}{1-r}-U_{W^{*}}(r, s)\right) .
\end{aligned}
$$

Now observe the following:

(i) the left-hand side of (33) is analytic in $\operatorname{Re} s>0$ and continuous in $\operatorname{Re} s \geq 0$,

(ii) the right-hand side of (33) is analytic in $\operatorname{Re} s<0$ and continuous in $\operatorname{Re} s \leq 0$,

(iii) for large $s$, both sides are $O\left(s^{l}\right)$ in their respective half-planes.

At the boundary $\operatorname{Re} s=0$, both sides are well defined, so that we again have a WienerHopf boundary value problem. As before, the $G(r, s)$ that is equal to the left-hand side of (33) for $\operatorname{Re} s \geq 0$ and to the right-hand side of (33) for $\operatorname{Re} s \leq 0$ is analytic in the whole $s$-plane, and $G(r, s)=O\left(s^{\ell}\right)$ for large $s$. According to Liouville's theorem both sides of (33), in their respective half-plane, are equal to the same $(\ell)$-th degree polynomial in $s$, i.e., for $\operatorname{Re} s \geq 0$,

$$
D_{B}(s)\left(U_{W}(r, s)-\mathrm{e}^{-s w}\right)=\sum_{k=0}^{\ell} a_{k}(r) s^{k}
$$

for $\operatorname{Re} s \geq 0$ and

$$
r N_{B}(s) \Phi_{A}(-s) U_{V W}(r, s)+r D_{B}(s)\left(\frac{1}{1-r}-U_{W^{*}}(r, s)\right)=\sum_{k=0}^{\ell} a_{k}(r) s^{k}
$$

for $\operatorname{Re} s \leq 0$. We still need to determine the $\ell+1$ unknown functions $a_{0}(r), \ldots, a_{\ell}(r)$. Taking $s=0$ in either (34) or (35) gives the expression in (32) for $a_{0}(r)$. Next we take $s=s_{j}, j=1, \ldots, \ell$. We do this in (35), observing that $\operatorname{Re} s_{j}<0$. Using that $D_{B}\left(s_{j}\right)=0$ we thus obtain

$$
r N_{B}\left(s_{j}\right) \Phi_{A}\left(-s_{j}\right) U_{V W}\left(r, s_{j}\right)=\sum_{k=0}^{\ell} a_{k}(r) s_{j}^{k}, \quad j=1, \ldots, \ell .
$$


Applying (6), this identity can be rewritten into

$$
r N_{B}\left(s_{j}\right) \Phi_{A}\left(-s_{j}\right) \int_{-\infty}^{0} U_{W}\left(r, s_{j} y\right) \mathbb{P}(V \in \mathrm{d} y)=\sum_{k=0}^{\ell} a_{k}(r) s_{j}^{k}, \quad j=1, \ldots, \ell .
$$

Using (34), Eq. (37) becomes, for $j=1, \ldots, \ell$,

$r N_{B}\left(s_{j}\right) \Phi_{A}\left(-s_{j}\right) \int_{-\infty}^{0}\left(\mathrm{e}^{-s_{j} y w}+\frac{\sum_{k=0}^{\ell} a_{k}(r)\left(s_{j} y\right)^{k}}{D_{B}\left(s_{j} y\right)}\right) \mathbb{P}(V \in \mathrm{d} y)=\sum_{k=0}^{\ell} a_{k}(r) s_{j}^{k}$.

We can rewrite this equation as follows: for $j=1, \ldots, \ell$,

$$
\begin{aligned}
& \sum_{k=0}^{\ell} a_{k}(r) s_{j}^{k}\left(1-r N_{B}\left(s_{j}\right) \Phi_{A}\left(-s_{j}\right) \int_{-\infty}^{0} \frac{y^{k}}{\prod_{m=1}^{\ell}\left(s_{j} y-s_{m}\right)} \mathbb{P}(V \in \mathrm{d} y)\right) \\
& \quad=r N_{B}\left(s_{j}\right) \Phi_{A}\left(-s_{j}\right) \Phi_{V}\left(s_{j} w\right) .
\end{aligned}
$$

One can determine the remaining unknowns $a_{1}(r), \ldots, a_{\ell}(r)$ from this set of $\ell$ linear equations. Subsequently, from (34), (31) follows.

It should be observed that the function $U_{W^{*}}$ can be obtained from (31) and (35).

If $\mathbb{P}(B \leq A)>0$ holds then condition (C1) is fulfilled, so $W_{n}$ weakly converges to a proper limit. We obtain the steady-state behavior via an Abelian theorem for power series:

$$
\Phi_{W}(s)=\lim _{r \uparrow 1}(1-r) U_{W}(r, s)=\frac{\sum_{k=0}^{\ell} a_{k} s^{k}}{D_{B}(s)}, \quad \operatorname{Re} s \geq 0
$$

where $a_{k}:=\lim _{r \uparrow 1}(1-r) a_{k}(r)$, for $k=0, \ldots, \ell$. Using (32) and (39), we readily obtain the linear system

$$
\sum_{k=0}^{\ell} a_{k} s_{j}^{k}\left(1-N_{B}\left(s_{j}\right) \Phi_{A}\left(-s_{j}\right) \int_{-\infty}^{0} \frac{y^{k}}{\prod_{m=1}^{\ell}\left(s_{j} y-s_{m}\right)} \mathbb{P}(V \in \mathrm{d} y)\right)=0
$$

for $a_{j}, j=1, \ldots, \ell$, while $a_{0}=(-1)^{\ell} \prod_{i=1}^{\ell} s_{i}$.

The mean of $W$ directly follows by differentiation of (40): $\Phi_{W}^{\prime}(0)=\left(a_{1} D_{B}(0)-\right.$ $\left.a_{0} D_{B}^{\prime}(0)\right) / D_{B}(0)^{2}$, with $D_{B}^{\prime}(0)=1$ if $\ell=1$ and $D_{B}^{\prime}(0)=-\sum_{i=1}^{\ell} s_{i}$ if $\ell=2,3, \ldots$; hence,

$$
\mathbb{E}(W)= \begin{cases}\frac{1-a_{1}}{a_{0}}, & \ell=1 ; \\ -\frac{a_{1}+\sum_{i=1}^{\ell} s_{i}}{a_{0}}, & \ell=2,3, \ldots\end{cases}
$$


Remark 1 It follows from (40) that $W$ is a mixture of an atom at zero (with probability $\left.a_{\ell}\right)$ and $\ell$ exponential terms. This is not surprising: as $V_{i}<0$, the only way for $W_{i+1}$ to be positive is to have $B_{i}>A_{i}-V_{i} W_{i}$. Now use the fact that $B_{i}$ has a phase-type distribution with $\ell$ exponential phases, in combination with the memoryless property of the exponential distribution.

Remark 2 When $\ell=1$, one obtains, using that $a_{0}=-s_{1}$,

$$
a_{1}=\frac{1-N_{B}\left(s_{1}\right) \Phi_{A}\left(-s_{1}\right) \int_{-\infty}^{0} \frac{1}{y-1} \mathbb{P}(V \in \mathrm{d} y)}{1-N_{B}\left(s_{1}\right) \Phi_{A}\left(-s_{1}\right)\left(\int_{-\infty}^{0} \frac{1}{y-1} \mathbb{P}(V \in \mathrm{d} y)+1\right)}
$$

For general $\ell$, we have not been able to verify formally that the set of $\ell$ linear equations (41) in the $\ell$ unknowns $a_{1}, \ldots, a_{\ell}$ has a unique solution (as they involve the zeroes $s_{j}$ and the distribution of $V$ in an intricate way); similarly for the set of equations (39) for $a_{1}(r), \ldots, a_{\ell}(r)$. However, since $W_{i}$ has a unique limiting distribution with $\operatorname{LST} \Phi_{W}(s)$ as $i \rightarrow \infty$, there is no reason to suspect that anomalies in this set of equations will occur.

Example 1 Suppose that $B$ has an exponential distribution with mean $1 / \mu$. Then $\Phi_{B}(s)=\mu /(s+\mu), \ell=1$ and $s_{1}=-\mu$. Suppose also that $V=-a$ a.s. with $a>0$. We then obtain

$$
a_{0}(r)=\frac{r \mu}{1-r}, \quad a_{1}(r)=\frac{r}{1-r}\left(1-\frac{(1+a) r \Phi_{A}(\mu)}{1+a+\operatorname{ar} \Phi_{A}(\mu)}\right)-\frac{(1+a) r \Phi_{A}(\mu)}{1+a+\operatorname{ar} \Phi_{A}(\mu)} e^{-a \mu w} .
$$

Multiplying with $(1-r)$ and letting $r \uparrow 1$ yields the coefficients

$$
a_{0}=\mu, \quad a_{1}=1-\frac{(1+a) \Phi_{A}(\mu)}{1+a+a \Phi_{A}(\mu)},
$$

so that the LST of $W$ is given by

$$
\Phi_{W}(s)=\frac{a_{0}+a_{1} s}{\mu+s}=\mathbb{P}(W>0) \frac{\mu}{\mu+s}+\mathbb{P}(W=0),
$$

where the last equality follows from $\mathbb{P}(W=0)=\lim _{s \rightarrow \infty} \Phi_{W}(s)=a_{1}$. We then obtain $\mathbb{E}(W)=\left(1-a_{1}\right) / \mu$ in accordance with (42).

The case where $a=1$, yielding the Lindley-type recursion $W_{i+1}=\left[B_{i}-A_{i}-W_{i}\right]^{+}$, has been extensively studied in [23]. We obtain for the stationary process

$$
a_{0}=\mu, \quad a_{1}=\frac{2-\Phi_{A}(\mu)}{2+\Phi_{A}(\mu)},
$$

which is in agreement with [23, Formula (4.12), p. 74]. It is easy to see that $\mathbb{P}(W=$ $0)=a_{1}$ is increasing in $a$.

For $a=0$, we have $\mathbb{P}(W=0)=1-\Phi_{A}(\mu)$. This relation is explained by observing that now $\mathbb{P}(W=0)=\mathbb{P}(B<A)$, with $B \sim \exp (\mu)$. For $a \uparrow \infty$ we have 
$\mathbb{P}(W=0)=1 /\left(1+\Phi_{A}(\mu)\right)$, which is explained by observing that a positive $W$ is followed by a geometric $(q)$ number of zeroes, with $q=\mathbb{P}(B<A)=1-\Phi_{A}(\mu)$.

\section{Model III: the uniform proportional case}

In this section, we once more consider the stochastic recursion $W_{i+1}=\left[V_{i} W_{i}+Y_{i}\right]^{+}$, where $Y_{i}=B_{i}-A_{i}$. Again we impose the usual independence assumptions on the sequences $\left\{V_{i}\right\}_{i \in \mathbb{N}_{0}},\left\{A_{i}\right\}_{i \in \mathbb{N}_{0}}$, and $\left\{B_{i}\right\}_{i \in \mathbb{N}_{0}}$. In addition, we assume that the $A_{i}$ are $\exp (\lambda)$ distributed. The 'multiplicative adjustments' $\left\{V_{i}\right\}_{i \in \mathbb{N}_{0}}$ are assumed to form a sequence of unit uniformly distributed random variables on $[0,1]$. By Theorem 2 , since $\mathbb{E}(\log |V|)<0$, a steady-state distribution of $\left\{W_{i}\right\}_{i \in \mathbb{N}_{0}}$ always exists. We shall first study its transient distribution and then obtain the steady-state distribution.

We start with (8), i.e.,

$$
\Phi_{W_{i+1}}(s)=\Phi_{V_{i} W_{i}+B_{i}-A_{i}}(s)+1-\Phi_{W_{i}^{*}}(s), \quad i=0,1, \ldots,
$$

where as before $W_{i}^{*}=\left[V_{i} W_{i}+B_{i}-A_{i}\right]^{-}$. This time the distribution of $W_{i}^{*}$ is almost trivial: either $V_{i} W_{i}+B_{i}-A_{i} \geq 0$, in which case $W_{i}^{*}=0$, or $W_{i}^{*}$ has the same exponential distribution as $A_{i}$, due to the lack of memory property of the exponential distribution.

Using the independence between $\left\{A_{i}\right\}_{i \in \mathbb{N}_{0}},\left\{B_{i}\right\}_{i \in \mathbb{N}_{0}}$, and $\left\{V_{i}\right\}_{i \in \mathbb{N}_{0}}$ and the exponentiality of the $A_{i}$, we obtain

$$
\begin{aligned}
\Phi_{W_{i+1}}(s)= & \Phi_{V_{i} W_{i}}(s) \Phi_{B}(s) \frac{\lambda}{\lambda-s}+1-\mathbb{P}\left(V_{i} W_{i}+B_{i}-A_{i}<0\right) \frac{\lambda}{\lambda-s} \\
& -\mathbb{P}\left(V_{i} W_{i}+B_{i}-A_{i} \geq 0\right) \\
= & \Phi_{V_{i} W_{i}}(s) \Phi_{B}(s) \frac{\lambda}{\lambda-s}-p_{i+1} \frac{s}{\lambda-s},
\end{aligned}
$$

where we set $p_{i}:=\mathbb{P}\left(W_{i}=0\right)$. Our goal is to write (44) fully in terms of the functions $\Phi_{W_{i}}(s)$. To this end, performing the change of variable $v:=s u$, we obtain

$$
\Phi_{V_{i} W_{i}}(s)=\int_{0}^{1} \mathbb{E}\left(\mathrm{e}^{-s u W_{i}}\right) \mathrm{d} u=\frac{1}{s} \int_{0}^{s} \Phi_{W_{i}}(v) \mathrm{d} v
$$

By multiplying with $\lambda-s$, we thus obtain the following recursive integral equation.

Lemma 7 For $i \in \mathbb{N}$,

$$
\Phi_{W_{i+1}}(s)=\frac{\lambda \Phi_{B}(s)}{s(\lambda-s)} \int_{0}^{s} \Phi_{W_{i}}(v) \mathrm{d} v-\frac{s}{\lambda-s} p_{i+1} .
$$

Since the LST $\Phi_{W_{0}}(s)=\mathrm{e}^{-s w}$ of $W_{0}$ is known, Relation (46) in principle allows us to recursively determine all the transforms $\Phi_{W_{i}}(\cdot), i \in \mathbb{N}$. Observe that, when $s=\lambda$, 
the right-hand side should become zero; using (45) we obtain

$$
p_{i+1}=\frac{\Phi_{B}(\lambda)}{\lambda} \int_{0}^{\lambda} \Phi_{W_{i}}(v) \mathrm{d} v
$$

This formula can easily be interpreted probabilistically, using the memoryless property of the exponential distribution for $A_{i}$ :

$$
\mathbb{P}\left(W_{i+1}=0\right)=P\left(A_{i} \geq B_{i}+V_{i} W_{i}\right)=\mathbb{P}\left(A_{i} \geq B_{i}\right) P\left(A_{i} \geq V_{i} W_{i}\right)=\Phi_{B}(\lambda) \Phi_{V_{i} W_{i}}(\lambda) .
$$

It is not possible to obtain explicit expressions for $\Phi_{W_{i}}$. However, as so often, one can utilize the method of generating functions to turn the recursion (46) into some sort of differential or integral equation. Therefore, we multiply Eq. (46) by $r^{i+1}$ and sum over $i$ to obtain

$$
U_{W}(r, s)=\frac{\lambda r \Phi_{B}(s)}{s(\lambda-s)} \int_{0}^{s} U_{W}(r, v) \mathrm{d} v+\kappa(s),
$$

where, to simplify the notation,

$$
\kappa(s)=\Phi_{W_{0}}(s)-\frac{s}{\lambda-s}\left(U_{W}(r, \infty)-p_{0}\right) .
$$

With $I(s)=\int_{0}^{s} U_{W}(r, v) \mathrm{d} v$, we obtain the linear first-order differential equation

$$
I^{\prime}(s)=\frac{\lambda r \Phi_{B}(s)}{s(\lambda-s)} I(s)+\kappa(s)
$$

As follows by standard techniques, this inhomogeneous differential equation is solved by

$$
I(s)=\exp \left(\lambda r \int_{c}^{s} \frac{\Phi_{B}(t)}{t(\lambda-t)} \mathrm{d} t\right)\left(\theta(c)+\int_{c}^{s} \kappa(u) \exp \left(-\lambda r \int_{c}^{u} \frac{\Phi_{B}(t)}{t(\lambda-t)} \mathrm{d} t\right) \mathrm{d} u\right),
$$

where necessarily $\theta(c)=I(c)$ and we assume for the time being that $c \in(s, \lambda)$ if $s<\lambda$ and $c \in(\lambda, s)$ if $\lambda<s$. Since $\Phi_{B}$ is bounded and bounded away from zero, we have as $s \downarrow \lambda$ (and likewise if $s \uparrow \lambda$ in the case where $s<\lambda$ ),

$$
\exp \left(\lambda r \int_{c}^{s} \frac{\Phi_{B}(t)}{t(\lambda-t)} \mathrm{d} t\right) \rightarrow \infty
$$

As a consequence, to make sure $U_{W}(r, \lambda)$ remains bounded,

$$
\theta(c)=-\int_{c}^{\lambda} \kappa(u) \exp \left(-\lambda r \int_{c}^{u} \frac{\Phi_{B}(t)}{t(\lambda-t)} \mathrm{d} t\right) \mathrm{d} u
$$


and therefore

$$
\begin{aligned}
I(s) & =\exp \left(\lambda r \int_{c}^{s} \frac{\Phi_{B}(t)}{t(\lambda-t)} \mathrm{d} t\right) \int_{\lambda}^{s} \kappa(u) \exp \left(-\lambda r \int_{c}^{u} \frac{\Phi_{B}(t)}{t(\lambda-t)} \mathrm{d} t\right) \mathrm{d} u \\
& =\int_{\lambda}^{s} \kappa(u) \exp \left(\lambda r \int_{u}^{s} \frac{\Phi_{B}(t)}{t(\lambda-t)} \mathrm{d} t\right) \mathrm{d} u .
\end{aligned}
$$

It is to be noted that the integrand in (52) tends to $\infty$ as $s \rightarrow \lambda$, which follows from the finiteness of $I(\lambda)$. Inserting (52) into (50), and recalling that $U_{W}(r, s)=I^{\prime}(s)$, yields the following expression for the generating function $U_{W}$ :

$$
U_{W}(r, s)=\kappa(s)+\frac{\lambda r \Phi_{B}(s)}{s(\lambda-s)} \int_{\lambda}^{s} \kappa(u) \exp \left(\lambda r \int_{u}^{s} \frac{\Phi_{B}(t)}{t(\lambda-t)} \mathrm{d} t\right) \mathrm{d} u .
$$

Plugging (49) into (53) we obtain

$$
\begin{aligned}
U_{W}(r, s)= & \kappa(s)+\frac{\lambda r \Phi_{B}(s)}{s(\lambda-s)} \int_{\lambda}^{s}\left(\Phi_{W_{0}}(u)-\frac{u\left(U_{W}(r, \infty)-p_{0}\right)}{\lambda-u}\right) \\
& \exp \left(\lambda r \int_{u}^{s} \frac{\Phi_{B}(t)}{t(\lambda-t)} \mathrm{d} t\right) \mathrm{d} u .
\end{aligned}
$$

It remains to determine $U_{W}(r, \infty)$. Keeping in mind that $U_{W}(r, s)-\kappa(s) \rightarrow 0$ by (49) we obtain, after some rearrangements,

$$
U_{W}(r, \infty)=p_{0}-\frac{\int_{\lambda}^{\infty} \Phi_{W_{0}}(u) \exp \left(-\lambda r \int_{u}^{\infty} \frac{\Phi_{B}(t)}{t(t-\lambda)} \mathrm{d} t\right) \mathrm{d} u}{\int_{\lambda}^{\infty} \frac{u}{u-\lambda} \exp \left(-\lambda r \int_{u}^{\infty} \frac{\Phi_{B}(t)}{t(t-\lambda)} \mathrm{d} t\right) \mathrm{d} u}
$$

We summarize our findings in the following theorem.

Theorem 8 For $r \in(0,1)$,

$$
U_{W}(r, s)=\kappa(s)+\frac{\lambda r \Phi_{B}(s)}{s(\lambda-s)} \int_{\lambda}^{s} \kappa(u) \exp \left(\lambda r \int_{u}^{s} \frac{\Phi_{B}(t)}{t(\lambda-t)} \mathrm{d} t\right) \mathrm{d} u
$$

where

$$
\kappa(s)=\Phi_{W_{0}}(s)+\frac{\frac{s}{\lambda-s} \int_{\lambda}^{\infty} \Phi_{W_{0}}(u) \exp \left(-\lambda r \int_{u}^{\infty} \frac{\Phi_{B}(t)}{t(t-\lambda)} \mathrm{d} t\right) \mathrm{d} u}{\int_{\lambda}^{\infty} \frac{u}{u-\lambda} \exp \left(-\lambda r \int_{u}^{\infty} \frac{\Phi_{B}(t)}{t(t-\lambda)} \mathrm{d} t\right) \mathrm{d} u} .
$$

The complexity of this type of result is comparable to that of recently studied related models; compare the structure of (54) with that of the transform of the transient storage level in, for example, [5].

We already noted that since the $V_{i}$ are uniformly distributed on $[0,1]$ we have $\mathbb{E}(\log |V|)<0$. Hence, we always have $W_{i} \Rightarrow W$ as $i \rightarrow \infty$ for some proper random variable $W$. Its LST is given in the following theorem. 
Theorem $9 W_{i}$ converges weakly to a proper limit $W$ as $i \rightarrow \infty$, and

$$
\Phi_{W}(s)=\frac{p_{\infty}}{s-\lambda}\left(s-\frac{\lambda \Phi_{B}(s)}{s} \int_{\lambda}^{s} \frac{u}{u-\lambda} \exp \left(\lambda \int_{u}^{s} \frac{\Phi_{B}(t)}{t(\lambda-t)} \mathrm{d} t\right) \mathrm{d} u\right),
$$

where

$$
p_{\infty}=\left[\int_{0}^{\lambda} \frac{1}{\lambda-u} \exp \left(-\int_{0}^{u}\left(\frac{\lambda \Phi_{B}(t)}{t(\lambda-t)}-\frac{1}{t}\right) \mathrm{d} t\right) \mathrm{d} u\right]^{-1} .
$$

Proof We apply an Abelian theorem and obtain (55) after multiplying both sides of (54) by $1-r$ and letting $r$ tend to one. We thereby use the fact that

$$
\lim _{r \uparrow 1}(1-r) \kappa(s)=\frac{s p_{\infty}}{s-\lambda} .
$$

The relation for $p_{\infty}$ follows by noting that $\Phi_{W}(0)=1$, so that

$$
\begin{aligned}
\frac{1}{p_{\infty}} & =\lim _{s \downarrow 0} \frac{1}{s-\lambda}\left(s-\frac{\lambda \Phi_{B}(s)}{s} \int_{\lambda}^{s} \frac{u}{u-\lambda} \exp \left(\lambda \int_{u}^{s} \frac{\Phi_{B}(t)}{t(\lambda-t)} \mathrm{d} t\right) \mathrm{d} u\right) \\
& =\lim _{s \downarrow 0} \frac{1}{s} \int_{s}^{\lambda} \frac{u}{\lambda-u} \exp \left(-\lambda \int_{s}^{u} \frac{\Phi_{B}(t)}{t(\lambda-t)} \mathrm{d} t\right) \mathrm{d} u \\
& =\lim _{s \downarrow 0} \int_{s}^{\lambda} \frac{1}{\lambda-u} \exp \left(-\int_{s}^{u}\left(\frac{\lambda \Phi_{B}(t)}{t(\lambda-t)}-\frac{1}{t}\right) \mathrm{d} t\right) \mathrm{d} u .
\end{aligned}
$$

Now,

$$
\frac{\lambda \Phi_{B}(t)}{t(\lambda-t)}-\frac{1}{t} \rightarrow \frac{1}{\lambda}-\mathbb{E}(B)
$$

as $t \downarrow 0$, so that we can safely let $s \downarrow 0$ and obtain the finite and nonzero limit (56).

Remark 3 The expected value $\mathbb{E}(W)$ can be expressed in terms of the parameters $\lambda$, $\mathbb{E}(B)$ and $p_{\infty}$ as follows: Letting $i \rightarrow \infty$ in (44) yields

$$
\Phi_{W}(s)=\Phi_{V W}(s) \Phi_{B}(s) \frac{\lambda}{\lambda-s}-\frac{s}{\lambda-s} p_{\infty}
$$

After a rearrangement of terms, this becomes

$$
p_{\infty}=\Phi_{W}(s)+\lambda \frac{1-\Phi_{W}(s)+\Phi_{V W+B}(s)-1}{s} .
$$

As $s \downarrow 0$ the right-hand side tends to $1+\lambda(\mathbb{E}(W)-\mathbb{E}(V W+B))$ and since $\mathbb{E}(V W+$ $B)=\mathbb{E}(V) \mathbb{E}(W)+\mathbb{E}(B)=\frac{1}{2} \mathbb{E}(W)+\mathbb{E}(B)$, we obtain

$$
\mathbb{E}(W)=2\left(\mathbb{E}(B)-\frac{1-p_{\infty}}{\lambda}\right)
$$


This yields the inequality $\mathbb{P}(W=0) \geq 1-\lambda \mathbb{E}(B)$, the value that one would get if $V_{i} \equiv 1$.

Example 2 If the $B_{i}$ have a rational LST, Expression (55) for $\Phi_{W}(s)$ and (56) for $p_{\infty}$ simplify considerably. For $\exp (\mu)$ distributed $B_{i}$, a partial fraction expansion yields

$$
\frac{u}{\lambda-u} \exp \left(\lambda \int_{u}^{s} \frac{\Phi_{B}(t)}{t(\lambda-t)} \mathrm{d} t\right)=\frac{s}{\lambda-u}\left(\frac{\lambda-u}{\lambda-s}\right)^{\frac{\mu}{\lambda+\mu}}\left(\frac{\mu+u}{\mu+s}\right)^{\frac{\lambda}{\lambda+\mu}} .
$$

For $s<\lambda$, the integral can be expressed in terms of the incomplete beta function $B(x, a, b)=\int_{0}^{x} u^{a-1}(1-u)^{b-1} \mathrm{~d} u$ :

$$
\begin{aligned}
& \int_{\lambda}^{s} \frac{s}{u-\lambda}\left(\frac{\lambda-u}{\lambda-s}\right)^{\frac{\mu}{\lambda+\mu}}\left(\frac{\mu+u}{\mu+s}\right)^{\frac{\lambda}{\lambda+\mu}} \mathrm{d} u \\
& \quad=\frac{s(\lambda+\mu)}{(\mu+s)^{\frac{\lambda}{\lambda+\mu}}(\lambda-s)^{\frac{\mu}{\lambda+\mu}} B\left(\frac{\lambda-s}{\lambda+\mu}, \frac{\mu}{\lambda+\mu}, 1+\frac{\lambda}{\lambda+\mu}\right) .}
\end{aligned}
$$

We then obtain

$$
\Phi_{W}(s)=p_{\infty} \cdot\left(\frac{\lambda \mu(\lambda+\mu) B\left(\frac{\lambda-s}{\lambda+\mu}, \frac{\mu}{\lambda+\mu}, 1+\frac{\lambda}{\lambda+\mu}\right)}{(\mu+s)^{1+\frac{\lambda}{\lambda+\mu}}(\lambda-s)^{1+\frac{\mu}{\lambda+\mu}}}-\frac{s}{\lambda-s}\right) .
$$

This leads to

$$
p_{\infty}=\frac{\mu^{\frac{\lambda}{\lambda+\mu}} \lambda^{\frac{\mu}{\lambda+\mu}}}{(\lambda+\mu) B\left(\frac{\lambda}{\lambda+\mu}, \frac{\mu}{\lambda+\mu}, 1+\frac{\lambda}{\lambda+\mu}\right)}
$$

so that, at least for $s<\lambda$,

$$
\begin{aligned}
\Phi_{W}(s)= & \frac{\mu^{\frac{\lambda}{\lambda+\mu}} \lambda^{\frac{\mu}{\lambda+\mu}}}{(\lambda+\mu) B\left(\frac{\lambda}{\lambda+\mu}, \frac{\mu}{\lambda+\mu}, 1+\frac{\lambda}{\lambda+\mu}\right)} \\
& \cdot\left(\frac{\lambda \mu(\lambda+\mu) B\left(\frac{\lambda-s}{\lambda+\mu}, \frac{\mu}{\lambda+\mu}, 1+\frac{\lambda}{\lambda+\mu}\right)}{\left.(\mu+s)^{1+\frac{\lambda}{\lambda+\mu}}(\lambda-s)^{1+\frac{\mu}{\lambda+\mu}}-\frac{s}{\lambda-s}\right) .} .\right.
\end{aligned}
$$

Unfortunately, a similar expression for the $s>\lambda$ case is not available. Instead, one obtains expressions that involve hypergeometric functions. Also it seems very hard to obtain higher moments from (59) by means of differentiation. It is possible, however, to derive a recursion formula for the moments $\omega_{k}:=\mathbb{E}\left(W^{k}\right)$ (where we assume their existence for $k=1,2, \ldots, j$, say) if we start with (57), which in our example becomes

$$
(\lambda-s) \Phi_{W}(s)=\frac{\lambda \mu}{s(\mu+s)} \int_{0}^{s} \Phi_{W}(v) \mathrm{d} v-s p_{\infty} .
$$


For $s<0$, the expansion $\Phi_{W}(-s)=\sum_{k=0}^{j} \frac{\omega_{k}}{k !} s^{k}+o\left(s^{j}\right)$ holds. Inserting this into (60) yields

$$
(\mu-s)(\lambda+s) \sum_{k=0}^{j} \frac{\omega_{k}}{k !} s^{k}+o\left(s^{j}\right)=\lambda \mu \sum_{k=0}^{j} \frac{\omega_{k}}{(k+1) !} s^{k}-s(\mu+s) p_{\infty}+o\left(s^{j}\right), \quad s \downarrow 0 .
$$

Equating the coefficients on both sides leads to

$$
\begin{aligned}
& \lambda \mu \frac{\omega_{k}}{k !}+(\mu-\lambda) \frac{\omega_{k-1}}{(k-1) !}-\frac{\omega_{k-2}}{(k-2) !} \mathbb{1}_{\{k \geq 2\}} \\
& =\left(\lambda \mu \frac{\omega_{1}}{2}-\mu p_{\infty}\right) \mathbb{1}_{\{k=1\}}+\left(\lambda \mu \frac{\omega_{2}}{6}-p_{\infty}\right) \mathbb{1}_{\{k=2\}}+\frac{\lambda \mu \omega_{k}}{(k+1) !} \mathbb{1}_{\{k \geq 3\}}, \quad k \in\{1, \ldots, j\} .
\end{aligned}
$$

Then, in accordance with the general result (58),

$$
\omega_{1}=2\left(\frac{1}{\mu}-\frac{1-p_{\infty}}{\lambda}\right) .
$$

Moreover,

$$
\omega_{2}=3 \frac{1-p_{\infty}-(\mu-\lambda) \omega_{1}}{\mu \lambda}
$$

and

$$
\omega_{k}=\frac{\left(k^{2}-1\right) \omega_{k-2}-(k+1)(\mu-\lambda) \omega_{k-1}}{\lambda \mu}, \quad k \in\{3, \ldots, j\}
$$

Remark 4 One can generalize Model III to the case in which $V=U^{1 / \alpha}$, where $U$ has a uniform distribution on $[0,1]$ and $\alpha>0$. In this case (46) becomes

$$
s^{\alpha-1} \Phi_{W_{i+1}}(s)=\frac{\alpha \lambda \Phi_{B}(s)}{s(\lambda-s)} \int_{0}^{s} v^{\alpha-1} \Phi_{W_{i}}(v) \mathrm{d} v-\frac{s^{\alpha}}{(\lambda-s)} p_{i+1} .
$$

Letting $U_{W}^{(\alpha)}(r, s)=s^{\alpha-1} U_{W}(r, s)$ this yields

$$
U_{W}^{(\alpha)}(r, s)=\frac{\lambda r \alpha \Phi_{B}(s)}{s(\lambda-s)} \int_{0}^{s} U_{W}^{(\alpha)}(r, v) \mathrm{d} v+\kappa^{(\alpha)}(s),
$$

where

$$
\kappa^{(\alpha)}(s)=s^{\alpha-1} \Phi_{W_{0}}(s)-\frac{s^{\alpha}}{\lambda-s}\left(U_{W}^{(\alpha)}(r, \infty)-p_{0}\right) .
$$

Equation (61) is of the exact same type as (48), only with $r$ replaced by $r \alpha$. This allows one to derive $U_{W}^{(\alpha)}(r, s)$ in the same way as before. 


\section{Discussion and concluding remarks}

This paper has analyzed three reflected (or delayed at zero) autoregressive processes specified by the stochastic recursion $W_{i+1}=\left[V_{i} W_{i}+B_{i}-A_{i}\right]^{+}$. While the classical case of $V \equiv 1$ has been widely studied in the queueing literature, our more general setting allows explicit analysis only in special cases. The three special cases we have considered are: (i) $V$ equals a positive value $a$ with certain probability $p \in(0,1)$ and is negative otherwise, and both $A$ and $B$ have a rational LST, (ii) $V$ attains negative values only and $B$ has a rational LST, (iii) $V$ is uniformly distributed on $[0,1]$, and $A$ is exponentially distributed. In all three cases, we present transient and stationary results, where the transient results are in terms of the transform at a geometrically distributed epoch.

Cases which might allow explicit analysis are, for example:

1. A combination of Models II and III, allowing $V$ to be either negative or having a distribution as in Remark 4.

2. One might consider more general recursions, such as the high-order Lindley equations analyzed in, for example, [2,18,20].

Another possible line of research concerns scaling limits and asymptotics. In particular, tail asymptotics seem to be within reach; in heavy-tailed cases these may be identified relying on a Tauberian approach. One also anticipates that, under particular scalings, an explicit analysis is possible. Specifically, one would expect that a diffusion analysis similar to the one presented in [7] can be performed.

Acknowledgements The research of Boxma and Mandjes is partly funded by the NWO Gravitation Programme NETWORKS (Grant Number 024.002.003) and an NWO Top Grant (Grant Number 613.001.352). The research of Palmowski is partially supported by Polish National Science Centre Grant No. 2018/29/B/ST1/00756, 2019-2022.

Open Access This article is licensed under a Creative Commons Attribution 4.0 International License, which permits use, sharing, adaptation, distribution and reproduction in any medium or format, as long as you give appropriate credit to the original author(s) and the source, provide a link to the Creative Commons licence, and indicate if changes were made. The images or other third party material in this article are included in the article's Creative Commons licence, unless indicated otherwise in a credit line to the material. If material is not included in the article's Creative Commons licence and your intended use is not permitted by statutory regulation or exceeds the permitted use, you will need to obtain permission directly from the copyright holder. To view a copy of this licence, visit http://creativecommons.org/licenses/by/4.0/.

\section{References}

1. Asmussen, S.: Applied Probability and Queues. Springer, Berlin (2008)

2. Biggins, J.: Lindley-type equations in the branching random walk. Stoch. Process. Appl. 75, 105-133 (1998)

3. Borovkov, A.A.: Ergodicity and Stability of Stochastic Processes. Wiley, New York (1998)

4. Borovkov, A.A., Foss, S.G.: Stochastically recursive sequences and their generalizations. Sib. Adv. Math. 2(1), 16-81 (1992)

5. Boxma, O., Mandjes, M.: Affine storage and insurance risk models. Math. Oper. Res. (2021) (to appear)

6. Boxma, O., Vlasiou, M.: On queues with service and interarrival times depending on waiting times. Queueing Syst. 56, 121-132 (2007) 
7. Boxma, O., Mandjes, M., Reed, J.: On a class of reflected AR(1) processes. J. Appl. Probab. 53(3), 818-832 (2016)

8. Boxma, O., Löpker, A., Mandjes, M.: On two classes of reflected autoregressive processes. J. Appl. Probab. 57(2), 657-678 (2020)

9. Brandt, A.: The stochastic equation $Y_{n+1}=A_{n} Y_{n}+B_{n}$ with stationary coefficients. Adv. Appl. Probab. 18(1), 211-220 (1986)

10. Cohen, J.W.: The Wiener-Hopf technique in applied probability. J. Appl. Probab. 12(S1), 145-156 (1975)

11. Cohen, J.W.: The Single Server Queue. North Holland, New York (1982)

12. Devroye, L.: Simulating perpetuities. Methodol. Comput. Appl. Probab. 3(1), 97-115 (2001)

13. Diaconis, P., Freedman, D.: Iterated random functions. SIAM Rev. 41(1), 45-76 (1999)

14. Embrechts, P., Goldie, C.: Perpetuities and random equations. In: Asymptotic Statistics, pp. 75-86. Springer, Berlin (1994)

15. Foss, S., Konstantopoulos, T.: An overview of some stochastic stability methods. J. Oper. Res. Soc. Jpn. 47(4), 275-303 (2004)

16. Foss, S., Shneer, V., Thomas, J.P., Worrall, T.: Stochastic stability of monotone economies in regenerative environments. J. Econ. Theory 173, 334-360 (2018)

17. Horst, U.: The stochastic equation $Y_{t+1}=A_{t} Y_{t}+B_{t}$ with non-stationary coefficients. J. Appl. Probab. 38(1), 80-94 (2001)

18. Karpelevich, F., Kelbert, M., Suhov, Y.: Higher-order Lindley equations. Stoch. Process. Appl. 53, 65-96 (1994)

19. Kesten, H.: Random difference equations and renewal theory for products of random matrices. Acta Math. 131, 207-248 (1973)

20. Olvera-Cravioto, M., Ruiz-Lacedelli, O.: Stationary waiting time in parallel queues with synchronization. Math. Oper. Res. 46(1), 1-27 (2021)

21. Titchmarsh, E.C.: The Theory of Functions. Oxford University Press, Oxford (1968)

22. Vervaat, W.: On a stochastic difference equation and a representation of non-negative infinitely divisible random variables. Adv. Appl. Probab. 11(4), 750-783 (1979)

23. Vlasiou, M.: Lindley-type recursions. Ph.D. thesis, Eindhoven University of Technology (2006)

24. Whitt, W.: Queues with service times and interarrival times depending linearly and randomly upon waiting times. Queueing Syst. 6(1), 335-351 (1990)

Publisher's Note Springer Nature remains neutral with regard to jurisdictional claims in published maps and institutional affiliations. 